\title{
A Toda bracket in the stable homotopy groups of spheres
}

\author{
XiUgui LiU
}

Let $p$ be a prime number greater than five. In the $p$-local stable homotopy groups of spheres, H Toda and J Lin, respectively, constructed the elements

$$
\begin{gathered}
\gamma_{s} \in \pi_{2 s p^{3}-2 p^{2}-2 p-2 s+1}(S), \\
\omega_{m, n} \in \pi_{2 p^{n+1}-2 p^{n}+2 p^{m+1-2 p^{m}+2 p-6}(S)}
\end{gathered}
$$

of order $p$. In this paper, we show the nontriviality of the Toda bracket $\left\langle\gamma_{s}, p, \omega_{m, n}\right\rangle$ in the stable homotopy groups of spheres, where $n \geqslant m+2>6,3 \leqslant s<p$.

$55 \mathrm{Q} 45,55 \mathrm{~T} 15 ; 55 \mathrm{~S} 10$

\section{Introduction}

We are interested in the problem of detecting nontrivial elements in the stable homotopy groups of spheres. So far, several methods have been found to determine the stable homotopy groups of spheres. For example we have the classical Adams spectral sequence (ASS) [1] based on the Eilenberg-MacLane spectrum $K \mathbb{Z}_{p}$, whose $E_{2}$-term is $\operatorname{Ext}_{A}^{s, t}\left(\mathbb{Z}_{p}, \mathbb{Z}_{p}\right)$ and Adams differential given by

$$
\tilde{d}_{r}: E_{r}^{s, t} \longrightarrow E_{r}^{s+r, t+r-1},
$$

where $A$ denotes the mod $p$ Steenrod algebra. We also have the Adams-Novikov spectral sequence (ANSS) (see Miller, Ravenel and Wilson [7] and Ravenel [8]) based on the Brown-Peterson spectrum $B P$.

Throughout the paper, we fix a prime $p \geqslant 7$, and put $q=2(p-1)$. From Liulevicius [6], $\operatorname{Ext}_{A}^{1, *}\left(\mathbb{Z}_{p}, \mathbb{Z}_{p}\right)$ has $\mathbb{Z}_{p}$-basis consisting of $a_{0} \in \operatorname{Ext}_{A}^{1,1}\left(\mathbb{Z}_{p}, \mathbb{Z}_{p}\right), h_{i} \in$ $\operatorname{Ext}_{A}^{1, p^{i} q}\left(\mathbb{Z}_{p}, \mathbb{Z}_{p}\right)$ for all $i \geqslant 0$ and $\operatorname{Ext}_{A}^{2, *}\left(\mathbb{Z}_{p}, \mathbb{Z}_{p}\right)$ has $\mathbb{Z}_{p}$-basis consisting of $\alpha_{2}$, $a_{0}^{2}, a_{0} h_{i}(i>0), g_{i}(i \geqslant 0), k_{i}(i \geqslant 0), b_{i}(i \geqslant 0)$ and $h_{i} h_{j}(j \geqslant i+2, i \geqslant 0)$ whose internal degrees are $2 q+1,2, p^{i} q+1, p^{i+1} q+2 p^{i} q, 2 p^{i+1} q+p^{i} q, p^{i+1} q$ and $p^{i} q+p^{j} q$, respectively.

Let $M$ be the Moore spectrum modulo the prime $p$ given by the cofibration

$$
S \stackrel{p}{\rightarrow} S \stackrel{i}{\rightarrow} M \stackrel{j}{\rightarrow} \Sigma S,
$$


where $S$ is the sphere spectrum localized at the prime $p$. Let $\alpha: \Sigma^{q} M \rightarrow M$ be the Adams map and $V(1)$ be its cofibre given by the cofibration

$$
\Sigma^{q} M \stackrel{\alpha}{\rightarrow} M \stackrel{i^{\prime}}{\rightarrow} V(1) \stackrel{j^{\prime}}{\rightarrow} \Sigma^{q+1} M .
$$

This spectrum $V(1)$ is the known Toda-Smith spectrum. Let $V(2)$ be the cofibre of the $v_{2}$-map $\beta: \Sigma^{(p+1) q} V(1) \rightarrow V(1)$ given by the cofibration

$$
\Sigma^{(p+1) q} V(1) \stackrel{\beta}{\rightarrow} V(1) \stackrel{\bar{i}}{\rightarrow} V(2) \stackrel{\bar{j}}{\rightarrow} \Sigma^{(p+1) q+1} V(1) .
$$

Let $\gamma: \Sigma^{q\left(p^{2}+p+1\right)} V(2) \rightarrow V(2)$ be the $v_{3}$-map.

Definition 1.1 We define, for $t \geqslant 1$, the $\beta$-element $\beta_{t}=j^{\prime} j \beta^{t} i^{\prime} i \in \pi_{q[t p+(t-1)]-2}(S)$ and the $\gamma$-element $\gamma_{t}=j j^{\prime} \bar{j} \gamma^{t} \bar{i} i^{\prime} i \in \pi_{q\left[t p^{2}+(t-1) p+(t-2)\right]-3}(S)$. Here the maps $i$, $i^{\prime}, \bar{i}, \beta, j, j^{\prime}, \bar{j}$ and $\gamma$ are given above.

Theorem 1.2 With notation as above, we have:

(1) (Smith [9]) For $p \geqslant 5$ and $t \geqslant 1, \beta_{t} \neq 0$ in $\pi_{*}(S)$.

(2) (Toda [10]) For $p \geqslant 7$ and $t \geqslant 1, \gamma_{t} \neq 0$ in $\pi_{*}(S)$.

In [2], R Cohen constructed a certain infinite family denoted by $\zeta_{k} \in \pi_{q\left(p^{k+1}+1\right)-3}(S)$, $k \geqslant 1$. $\zeta_{k}$ is represented by

$$
h_{0} b_{k} \in \operatorname{Ext}_{A}^{3, q\left(p^{k+1}+1\right)}\left(\mathbb{Z}_{p}, \mathbb{Z}_{p}\right)
$$

in the ASS.

Using the method of ANSS, C-N Lee [3] proved that $\beta_{1}^{p-1} \zeta_{k}$ is nontrivial for all $k$, ie,

$$
b_{0}^{p-1} h_{0} b_{k}
$$

is a permanent cycle in the ASS and converges nontrivially to

$$
\beta_{1}^{p-1} \zeta_{k}
$$

This result gives another infinite family of homotopy elements in the stable homotopy groups of spheres.

In [4], J Lin constructed a new nontrivial element, called $\omega_{m, n}$, in $\pi_{q\left(p^{n}+p^{m}+1\right)-4}(S)$ of order $p$, which is represented by

$$
h_{0}\left(h_{m} b_{n-1}-h_{n} b_{m-1}\right) \in \operatorname{Ext}_{A}^{4, q\left(p^{n}+p^{m}+1\right)}\left(\mathbb{Z}_{p}, \mathbb{Z}_{p}\right)
$$

in the ASS. On the way to proving the main result, he detected a new family in the stable homotopy groups of $M$ and gave the following theorem. 
Theorem 1.3 [4] Let $p \geqslant 7, n \geqslant m+2 \geqslant 4$ and $h_{n} \in \operatorname{Ext}_{A}^{1, p^{n} q}\left(\mathbb{Z}_{p}, \mathbb{Z}_{p}\right)$. Then

$$
(i)_{*}\left(h_{0} h_{n} h_{m}\right) \in \operatorname{Ext}_{A}^{3, q\left(p^{n}+p^{m}+1\right)}\left(H^{*} M, \mathbb{Z}_{p}\right)
$$

is a permanent cycle in the ASS and converges to a nontrivial element

$$
\xi_{m, n} \in \pi_{q\left(p^{n}+p^{m}+1\right)-3}(M) .
$$

In [5], X Liu obtained the following theorem.

Theorem 1.4 [5] Let $p \geqslant 7,0 \leqslant s<p-3$. Then there exists the third Greek letter element

$$
\tilde{\gamma}_{s+3} \in \operatorname{Ext}_{A}^{s+3, q\left[(s+3) p^{2}+(s+2) p+(s+1)\right]+s}\left(\mathbb{Z}_{p}, \mathbb{Z}_{p}\right),
$$

and $\tilde{\gamma}_{s+3}$ converges to the $\gamma$-element

$$
\gamma_{s+3} \in \pi_{q\left[(s+3) p^{2}+(s+2) p+(s+1)\right]-3}(S)
$$

in the ASS.

In this paper, I will use the new family of homotopy elements in $\pi_{*}(M)$ in [4] to detect a $\xi_{m, n}$-related family of filtration $s+6$ in the stable homotopy groups of spheres.

Theorem 1.5 Let $p \geqslant 7, n \geqslant m+2>6$ and $0 \leqslant s<p-3$. Then the product

$$
h_{0} h_{n} h_{m} \tilde{\gamma}_{s+3} \in \operatorname{Ext}_{A}^{s+6, t(s)}\left(\mathbb{Z}_{p}, \mathbb{Z}_{p}\right)
$$

is a permanent cycle in the ASS and converges to a nontrivial family of homotopy elements

$$
j j^{\prime} \bar{j} \gamma^{s+3} \bar{i}^{\prime} \xi_{m, n} \in \pi_{t(s)-s-6}(S),
$$

where $t(s)=q\left[p^{n}+p^{m}+(s+3) p^{2}+(s+2) p+(s+2)\right]+s$.

As the referee told me, in fact I show the nontriviality of Toda bracket $\left\langle\gamma_{s+3}, p, \omega_{m, n}\right\rangle$ in the stable homotopy groups of spheres and give the following theorem.

Theorem 1.6 Let $p \geqslant 7, n \geqslant m+2>6$ and $0 \leqslant s<p-3$. Then the Toda bracket

$$
\left\langle\gamma_{s+3}, p, \omega_{m, n}\right\rangle \subset \pi_{t(s)-s-6}(S)
$$

is essential. Here, $t(s)=q\left[p^{n}+p^{m}+(s+3) p^{2}+(s+2) p+(s+2)\right]+s$.

The May spectral sequence (MSS) and the ASS play very important roles in the proofs of the main results. The proof of our theorem is completely elementary.

The paper is arranged as follows: after giving some propositions on the MSS in Section 2, we will make use of the MSS to obtain two low-dimensional Ext groups in Section 3. Section 4 is devoted to showing Theorem 1.5. 


\section{The May spectral sequence (MSS)}

For computing the stable homotopy groups of spheres with the ASS, we must compute the $E_{2}$-term of the ASS, $\operatorname{Ext}_{A}^{*, *}\left(\mathbb{Z}_{p}, \mathbb{Z}_{p}\right)$. The most successful method for computing $\operatorname{Ext}_{A}^{*, *}\left(\mathbb{Z}_{p}, \mathbb{Z}_{p}\right)$ is the MSS.

From [8], there is a May spectral sequence (MSS) $\left\{E_{r}^{s, t, *}, d_{r}\right\}$ which converges to $\mathrm{Ext}_{A}^{s, t}\left(\mathbb{Z}_{p}, \mathbb{Z}_{p}\right)$ with $E_{1}$-term

(2.1) $E_{1}^{*, *, *}=E\left(h_{m, i} \mid m>0, i \geqslant 0\right) \otimes P\left(b_{m, i} \mid m>0, i \geqslant 0\right) \otimes P\left(a_{n} \mid n \geqslant 0\right)$,

where $E$ is the exterior algebra, $P$ is the polynomial algebra, and

$$
h_{m, i} \in E_{1}^{1,2\left(p^{m}-1\right) p^{i}, 2 m-1}, b_{m, i} \in E_{1}^{2,2\left(p^{m}-1\right) p^{i+1}, p(2 m-1)}, a_{n} \in E_{1}^{1,2 p^{n}-1,2 n+1} \text {. }
$$

One has

$$
d_{r}: E_{r}^{s, t, u} \rightarrow E_{r}^{s+1, t, u-r}
$$

and if $x \in E_{r}^{s, t, *}$ and $y \in E_{r}^{s^{\prime}, t^{\prime}, *}$, then

$$
d_{r}(x \cdot y)=d_{r}(x) \cdot y+(-1)^{s} x \cdot d_{r}(y) .
$$

There exists a graded commutativity in the MSS:

$$
x \cdot y=(-1)^{s s^{\prime}+t t^{\prime}} y \cdot x
$$

for $x, y=h_{m, i}, b_{m, i}$ or $a_{n}$. The first May differential $d_{1}$ is given by

$$
\left\{\begin{aligned}
d_{1}\left(h_{i, j}\right) & =\sum_{0<k<i} h_{i-k, k+j} h_{k, j}, \\
d_{1}\left(a_{i}\right) & =\sum_{0 \leqslant k<i} h_{i-k, k} a_{k}, \\
d_{1}\left(b_{i, j}\right) & =0 .
\end{aligned}\right.
$$

For each element $x \in E_{1}^{s, t, *}$, we $\operatorname{define} \operatorname{dim} x=s, \operatorname{deg} x=t$. Then we have

$$
\left\{\begin{aligned}
\operatorname{dim} h_{i, j} & =\operatorname{dim} a_{i}=1, \\
\operatorname{dim} b_{i, j} & =2, \\
\operatorname{deg} a_{0} & =1, \\
\operatorname{deg} h_{i, j} & =q\left(p^{i+j-1}+\cdots+p^{j}\right), \\
\operatorname{deg} b_{i, j} & =q\left(p^{i+j}+\cdots+p^{j+1}\right), \\
\operatorname{deg} a_{i} & =q\left(p^{i-1}+\cdots+p+1\right)+1,
\end{aligned}\right.
$$

where $i \geqslant 1, j \geqslant 0$. 
By the knowledge on the $p$-adic expression in number theory, we see that for each integer $t \geqslant 0$, it can be expressed uniquely as

$$
t=q\left(c_{n} p^{n}+c_{n-1} p^{n-1}+\cdots+c_{1} p+c_{0}\right)+e,
$$

where $0 \leqslant c_{i}<p(0 \leqslant i<n), p>c_{n}>0,0 \leqslant e<q$.

Theorem 2.3 [5] With notation as above, let $s_{1}$ be a positive integer with $0<s_{1}<p$. If $s_{1}<c_{j}$ for some $0 \leqslant j \leqslant n$, then in the MSS, we have that the $\mathbb{Z}_{p}$-module

$$
E_{1}^{s_{1}, t, *}=0 .
$$

Let $s_{2}$ and $t^{\prime}$ be two arbitrary positive integers. Suppose

$$
t^{\prime}=q\left(c_{n}^{\prime} p^{n}+c_{n-1}^{\prime} p^{n-1}+\cdots+c_{1}^{\prime} p+c_{0}^{\prime}\right)+e^{\prime},
$$

where $0 \leqslant c_{i}^{\prime}<p(0 \leqslant i<n), p>c_{n}^{\prime}>0,0 \leqslant e^{\prime}<q$. Suppose a generator of $E_{1}^{s_{2}, t^{\prime}, *}$ is of the form $h=x_{1} x_{2} \cdots x_{s_{3}} \in E_{1}^{s_{2}, t^{\prime}, *}$, where $x_{i}$ is one of $a_{k}, h_{r, j}$ or $b_{u, z}, 0 \leqslant k \leqslant n+1,0 \leqslant r+j \leqslant n+1,0 \leqslant u+z \leqslant n, r>0, j \geqslant 0, u>0, z \geqslant 0$. By (2.2) we can assume

$$
\operatorname{deg} x_{i}=q\left(c_{i, n} p^{n}+\cdots+c_{i, 1} p+c_{i, 0}\right)+e_{i},
$$

where $c_{i, j}=0$ or $1, e_{i}=1$ if $x_{i}=a_{k_{i}}$, or $e_{i}=0$. Then we have

$$
\operatorname{deg} h=\sum_{i=1}^{s_{3}} \operatorname{deg} x_{i}=q\left(\left(\sum_{i=1}^{s_{3}} c_{i, n}\right) p^{n}+\cdots+\left(\sum_{i=1}^{s_{3}} c_{i, 1}\right) p+\sum_{i=1}^{s_{3}} c_{i, 0}\right)+\sum_{i=1}^{s_{3}} e_{i} .
$$

Denote

by $\bar{c}_{j}$ and $\bar{e}, 0 \leqslant j \leqslant n$, respectively.

$$
\sum_{i=1}^{s_{3}} c_{i, j} \quad \text { and } \quad \sum_{i=1}^{s_{3}} e_{i}
$$

Theorem 2.4 With notation as above. Suppose that there exists some $0<j \leqslant n$ such that $\overline{c_{j}}=s_{3}$.

(1) If there also exist two integers $i_{1}$ and $i_{2}$ such that $0 \leqslant i_{1}<i_{2}<j$ and $s_{3} \geqslant$ $\bar{c}_{i_{1}}>\bar{c}_{i_{2}}$, then $h$ cannot exist.

(2) If there also exists an integer $i$ such that $0 \leqslant i<j$ and $s_{3} \geqslant \bar{e}>\bar{c}_{i}, h$ cannot exist.

(3) If there also exist two integers $i_{1}^{\prime}$ and $i_{2}^{\prime}$ such that $j<i_{1}^{\prime}<i_{2}^{\prime} \leqslant n$ and $s_{3} \geqslant$ $\bar{c}_{i_{2}^{\prime}}>\bar{c}_{i_{1}^{\prime}}$, then $h$ cannot exist.

Proof By (2.2), we easily get the desired result. 


\section{Application of the MSS to two Ext groups}

In this section, we make use of the MSS to determine two Ext groups which will be used in the proof of Theorem 1.5.

Lemma 3.1 Let $p \geqslant 7, n \geqslant m+2>6,0 \leqslant s<p-3$ and $r \geqslant 1$. Then the May $E_{1}$-term satisfies

$$
E_{1}^{s+6-r, t(s)+1-r, *}= \begin{cases}0 & r \geqslant 2 \\ 0 & r=1 \text { and } s<p-4 \\ M & r=1 \text { and } s=p-4 .\end{cases}
$$

Here $t(s)=q\left[p^{n}+p^{m}+(s+3) p^{2}+(s+2) p+(s+2)\right]+s$ and $M$ is the $\mathbb{Z}_{p}-$ module generated by nine elements

$$
\begin{aligned}
& \mathbf{g} i \in E_{1}^{p+1, t(p-4),(2 n+1) p-2 n-9} \quad(1 \leqslant i \leqslant 8) \\
& \mathbf{g} 9 \in E_{1}^{p+1, t(p-4),(2 m+1) p-2 m-9},
\end{aligned}
$$

where

$$
\begin{aligned}
& \mathbf{g} 1=a_{n}^{p-4} h_{n, 0} h_{4,0} h_{n-2,2} h_{n-3,3} h_{1, m}, \\
& \text { g2 }=a_{n}^{p-4} h_{n, 0} h_{m+1,0} h_{n-2,2} h_{1,3} h_{n-m, m}, \\
& \text { g3 }=a_{n}^{p-4} h_{n, 0} h_{4,0} h_{n-2,2} h_{m-2,3} h_{n-m, m}, \\
& \text { g4 }=a_{n}^{p-4} h_{n, 0} h_{m+1,0} h_{2,2} h_{n-3,3} h_{n-m, m}, \\
& \text { g5 }=a_{n}^{p-4} h_{n, 0} h_{4,0} h_{m-1,2} h_{n-3,3} h_{n-m, m}, \\
& \text { g6 }=a_{n}^{p-5} a_{m+1} h_{n, 0} h_{4,0} h_{n-2,2} h_{n-3,3} h_{n-m, m}, \\
& \text { g7 }=a_{n}^{p-5} a_{4} h_{n, 0} h_{m+1,0} h_{n-2,2} h_{n-3,3} h_{n-m, m}, \\
& \text { g8 }=a_{n}^{p-4} h_{m+1,0} h_{4,0} h_{n-2,2} h_{n-3,3} h_{n-m, m}, \\
& \text { g9 }=a_{m}^{p-4} h_{m, 0} h_{4,0} h_{m-2,2} h_{m-3,3} h_{1, n} .
\end{aligned}
$$

Proof When $r \geqslant s+4$, we can easily show that in the MSS $E_{1}^{s+6-r, t(s)+1-r, *}=0$. Thus in the rest of the proof, we assume that $1 \leqslant r<s+4$. Consider $h=x_{1} x_{2} \cdots x_{l} \in$ $E_{1}^{s+6-r, t(s)-r+1, *}$ in the MSS, where $x_{i}$ is one of $a_{k}, h_{r, j}$ or $b_{u, z}, 0 \leqslant k \leqslant n+1$, $0 \leqslant r+j \leqslant n+1,0 \leqslant u+z \leqslant n, r>0, j \geqslant 0, u>0, z \geqslant 0$. Assume that

$$
\operatorname{deg} x_{i}=q\left(c_{i, n} p^{n}+c_{i, n-1} p^{n-1}+\cdots+c_{i, 1} p+c_{i, 0}\right)+e_{i},
$$

where $c_{i, j}=0$ or $1, e_{i}=1$ if $x_{i}=a_{k_{i}}$, or $e_{i}=0$. It follows that

$\operatorname{dim} h=\sum_{i=1}^{l} \operatorname{dim} x_{i}=s+6-r$ 


$$
\begin{aligned}
\operatorname{deg} h & =\sum_{i=1}^{l} \operatorname{deg} x_{i}=q\left[\left(\sum_{i=1}^{l} c_{i, n}\right) p^{n}+\cdots+\left(\sum_{i=1}^{l} c_{i, 1}\right) p+\left(\sum_{i=1}^{l} c_{i, 0}\right)\right]+\left(\sum_{i=1}^{l} e_{i}\right) \\
& =q\left[p^{n}+p^{m}+(s+3) p^{2}+(s+2) p+(s+2)\right]+(s+1-r) .
\end{aligned}
$$

Note that $\operatorname{dim} h_{i, j}=\operatorname{dim} a_{i}=1, \operatorname{dim} b_{i, j}=2,1 \leqslant r<s+4$ and $0 \leqslant s<p-3$. From $\operatorname{dim} h=\sum_{i=1}^{l} \operatorname{dim} x_{i}=s+6-r$ we have $l \leqslant s+6-r<p+3-r \leqslant p+2$.

We claim that $s+1-r \geqslant 0$. Otherwise, we would have $\sum_{i=1}^{l} e_{i} \leqslant l \leqslant p+1$. On the other hand, by $1 \leqslant r<s+4$ and $p \geqslant 7$, we would have $\sum_{i=1}^{l} e_{i}=q+(s-r+1)>$ $2 p-2-3 \geqslant p+2$ which contradicts $\sum_{i=1}^{l} e_{i} \leqslant l \leqslant p+1$. The claim is proved.

Using $0 \leqslant s+3, s+2, s+1-r<p$ and the knowledge on $p$-adic expression in number theory, we have

$$
\begin{cases}\sum_{i=1}^{l} e_{i}=s+1-r+\lambda_{-1} q, & \lambda_{-1} \geqslant 0 \\ \sum_{i=1}^{l} c_{i, 0}+\lambda_{-1}=s+2+\lambda_{0} p, & \lambda_{0} \geqslant 0 \\ \sum_{i=1}^{l} c_{i, 1}+\lambda_{0}=s+2+\lambda_{1} p, & \lambda_{1} \geqslant 0 \\ \sum_{i=1}^{l} c_{i, 2}+\lambda_{1}=s+3+\lambda_{2} p, & \lambda_{2} \geqslant 0 \\ \sum_{i=1}^{l} c_{i, 3}+\lambda_{2}=0+\lambda_{3} p, & \lambda_{3} \geqslant 0 \\ \sum_{i=1}^{l} c_{i, 4}+\lambda_{3}=0+\lambda_{4} p, & \lambda_{4} \geqslant 0 \\ \vdots & \vdots \\ \sum_{i=1}^{l} c_{i, m-1}+\lambda_{m-2}=0+\lambda_{m-1} p, & \lambda_{m-1} \geqslant 0 \\ \sum_{i=1}^{l} c_{i, m}+\lambda_{m-1}=1+\lambda_{m} p, & \lambda_{m} \geqslant 0 \\ \sum_{i=1}^{l} c_{i, m+1}+\lambda_{m}=0+\lambda_{m+1} p, & \lambda_{m+1} \geqslant 0 \\ \sum_{i=1}^{l} c_{i, n}+\lambda_{n-1}=1 . & \end{cases}
$$


From $e_{i}=0$ or $1, c_{i, j}=0$ or 1 , and $l \leqslant p+1$, we easily have that

$$
\left(\lambda_{-1}, \lambda_{0}, \lambda_{1}, \lambda_{2}\right)=(0,0,0,0) .
$$

Consider the fifth equality of (3.2) $\sum_{i=1}^{l} c_{i, 3}=0+\lambda_{3} p$. By $c_{i, 3}=0$ or 1 , and $l \leqslant p+1$, we get that $\lambda_{3}=0$ or 1 .

Case $1 \lambda_{3}=0$. We claim $\lambda_{4}=0$. If $\lambda_{4}=1$, we would have $\sum_{i=1}^{l} c_{i, 2}=s+3$, $\sum_{i=1}^{l} c_{i, 3}=0, \sum_{i=1}^{l} c_{i, 4}=p$. From $\sum_{i=1}^{l} c_{i, 2}=s+3$ and (2.2), there would be $s+3$ factors among $h$ such that $\operatorname{deg} x_{i}=q$ (higher terms on $p+p^{2}+$ lower terms on $p$ ) $+\delta_{i}$, where $\delta_{i}$ may equal 0 or 1 . Similarly, from $\sum_{i=1}^{l} c_{i, 4}=p$, there would be $p$ factors among $h$ such that $\operatorname{deg} x_{i}=q$ (higher terms on $p+p^{4}+$ lower terms on $p$ ) $+\delta_{i}$. Thus, by $l \leqslant p+1$ and (2.2), there would be at least $p+s+3-(p+1)=s+2$ factors in $h$ such that $\operatorname{deg} x_{i}=q$ (higher terms on $p+p^{4}+p^{3}+p^{2}+$ lower terms on $p$ ) $+\delta_{i}$. Thus we would have $\sum_{i=1}^{l} c_{i, 3} \geqslant s+2$ which contradicts $\sum_{i=1}^{l} c_{i, 3}=0$. The claim is proved.

By induction on $j$, we have

$$
\lambda_{j}=0 \quad(4 \leqslant j \leqslant n-1) .
$$

Then we have the following:

Subcase 1.1 If there are two factors $h_{1, n}$ and $h_{1, m}$ in $h$, then up to sign $h=h_{1, n} h_{1, m} \tilde{h}$ with $\widetilde{h} \in E_{1}^{s+4-r, q\left[(s+3) p^{2}+(s+2) p+(s+2)\right]+(s+1-r), * \text {. }}$

When $r=1$, by an argument similar to that used in the proof of Theorem 1.1 of [5], $E_{1}^{s+3, q\left[(s+3) p^{2}+(s+2) p+(s+2)\right]+s, *}=0$.

When $r \geqslant 2, E_{1}^{s+4-r, q\left[(s+3) p^{2}+(s+2) p+(s+2)\right]+(s+1-r), *}=0$ by Theorem 2.3 From the above discussion we have there cannot exist two factors $h_{1, n}$ and $h_{1, m}$ in $h$.

Similarly, we can show the following.

Subcase 1.2 There cannot exist two factors $h_{1, n}$ and $b_{1, m-1}$ in $h$.

Subcase 1.3 There cannot exist two factors $b_{1, n-1}$ and $h_{1, m}$ in $h$.

Subcase 1.4 There cannot exist two factors $b_{1, n-1}$ and $b_{1, m-1}$ in $h$.

Case $2 \lambda_{3}=1$. When $r \geqslant 3$, it is easy to see that $\lambda_{3}$ is impossible to equal 1 . Thus in the rest of the proof, we assume $r \leqslant 2$. From the sixth equality of (3.2), $\sum_{i=1}^{l} c_{i, 4}+1=\lambda_{4} p$, and $0 \leqslant \sum_{i=1}^{l} c_{i, 4} \leqslant l \leqslant p+1$, we can deduce that

$$
\lambda_{4}=1 \text {. }
$$


By induction on $j$, we have

$$
\lambda_{j}=1 \quad(4 \leqslant j \leqslant m-1) .
$$

Now consider the $(m+2)-$ nd equality of (3.2), $\sum_{i=1}^{l} c_{i, m}+1=1+\lambda_{m} p$. Noting that $0 \leqslant \sum_{i=1}^{l} c_{i, m} \leqslant l \leqslant p+1$, we have that $\lambda_{m}=0$ or 1 .

Subcase 2.1 $\lambda_{m}=1$. Consider the $(m+3)-$ rd equality of (3.2), $\sum_{i=1}^{l} c_{i, m+1}+1=$ $\lambda_{m+1} p$. Using $0 \leqslant \sum_{i=1}^{l} c_{i, m+1} \leqslant l \leqslant p+1$, we can have that

$$
\lambda_{m+1}=1 \text {. }
$$

By induction on $j$, we can show

$$
\lambda_{j}=1 \quad(m+1 \leqslant j \leqslant n-1) .
$$

Thus we have

$$
\left(\lambda_{-1}, \lambda_{0}, \lambda_{1}, \lambda_{2}, \lambda_{3}, \ldots, \lambda_{m}, \lambda_{m+1}, \ldots, \lambda_{n-1}\right)=(0,0,0,0,1, \ldots, 1,1, \ldots, 1) .
$$

From the fifth equality of (3.2), $\sum_{i=1}^{l} c_{i, 3}=p$, using $c_{i, 3}=0$ or 1 , we have that $l \geqslant p$. Note that $l \leqslant s+5$. Thus $s \geqslant p-5$. By $0 \leqslant s<p-3$, we have that $s$ may equal $p-5$ or $p-4$.

(i) When $s=p-4, h=x_{1} x_{2} \cdots x_{l} \in E_{1}^{p+2-r, t(p-4)+1-r, *}$. From the first equality of (3.2), $\sum_{i=1}^{l} e_{i}=p-3-r$ and (2.2), there exist $(p-3-r)$ factors among $h$ such that

$$
\operatorname{deg} x_{i}=q(\text { higher terms on } p)+1 .
$$

Similarly, from $\sum_{i=1}^{l} c_{i, n-1}=p-1$, there exist $(p-1)$ factors among $h$ such that

$$
\operatorname{deg} x_{i}=q\left(p^{n-1}+\text { lower terms on } p\right)+\delta_{i},
$$

where $\delta_{i}$ may equal 0 or 1 . Noting $l \leqslant p+1$ and (2.2), we have that there exist at least $(p-3-r)+(p-1)-(p+1)=p-5-r$ factors in $h$ such that

$$
\operatorname{deg} x_{i}=q\left(p^{n-1}+\cdots+p+1\right)+1,
$$

ie, there exist at least $(p-5-r) a_{n}$ 's among $h$. By the graded commutativity of $E_{1}^{*, *, *}$, we can let $h=a_{n}^{p-5-r} x_{p-4-r} \cdots x_{l}$. Then $h^{\prime}=x_{p-4-r} \cdots x_{l} \in E_{1}^{7, t \prime \prime, *}$, where $t^{\prime \prime}=t(p-4)-(p-5-r) \operatorname{deg} a_{n}$. From $\sum_{i=1}^{l} c_{i, 3}=p$, we have $l \geqslant p$. Recall that $l \leqslant p+1$. Thus $p \leqslant l \leqslant p+1$.

If $l=p$, then

and

$$
\begin{aligned}
h & =a_{n}^{p-5-r} x_{p-4-r} \cdots x_{p} \in E_{1}^{p+2-r, t(p-4)+1-r, *} \\
h^{\prime} & =x_{p-4-r} \cdots x_{p} \in E_{1}^{7, t^{\prime \prime}, *} .
\end{aligned}
$$


Note that $\sum_{i=p-4-r}^{p} c_{i, 3}=r+5, \sum_{i=p-4-r}^{p} c_{i, 4}=r+4$ and $\sum_{i=p-4-r}^{p} c_{i, m}=r+5$. By Theorem 2.4, we have $E_{1}^{7, t^{\prime \prime}, *}=0$. Thus in this case $h$ cannot exist.

If $l=p+1$, then we can see that in this case $r$ cannot equal 2 by $\operatorname{dim} h=p+2-r$ and $\operatorname{dim} x_{i}=1$ or 2 . Thus in this case $r$ must equal 1 . Then $h=a_{n}^{p-6} x_{p-5} \cdots x_{p+1} \in$ $E_{1}^{p+1, t(p-4), *}$ and $h^{\prime}=x_{p-5} \cdots x_{p+1} \in E_{1}^{7, t^{\prime \prime}, *}$. It is easy to get that $\operatorname{dim} x_{i}=1$ for $p-5 \leqslant i \leqslant p+1$, ie,

$$
h^{\prime} \in E\left(h_{m, i} \mid m>0, i \geqslant 0\right) \otimes P\left(a_{n} \mid n \geqslant 0\right),
$$

and there exist at least five factors in $h^{\prime}$ such that $\operatorname{deg} x_{i}=q\left(\right.$ higher terms on $p+p^{m}+p^{m-1}+\cdots+p^{4}+p^{3}+$ lower terms on $\left.p\right)+\delta_{i}$ by $\sum_{i=p-5}^{p+1} c_{i, 3}=6, \sum_{i=p-5}^{p+1} c_{i, m}=6$ and (2.2), where $\delta_{i}$ may equal 0 or 1 . We can divide the seven factors of $h^{\prime}$ into the following three disjoint classes, using $\sum_{i=p-5}^{p+1} c_{i, 3}=6, \sum_{i=p-5}^{p+1} c_{i, 4}=5, \ldots, \sum_{i=p-5}^{p+1} c_{i, m-1}=5, \sum_{i=p-5}^{p+1} c_{i, m}=6$ and (2.2):

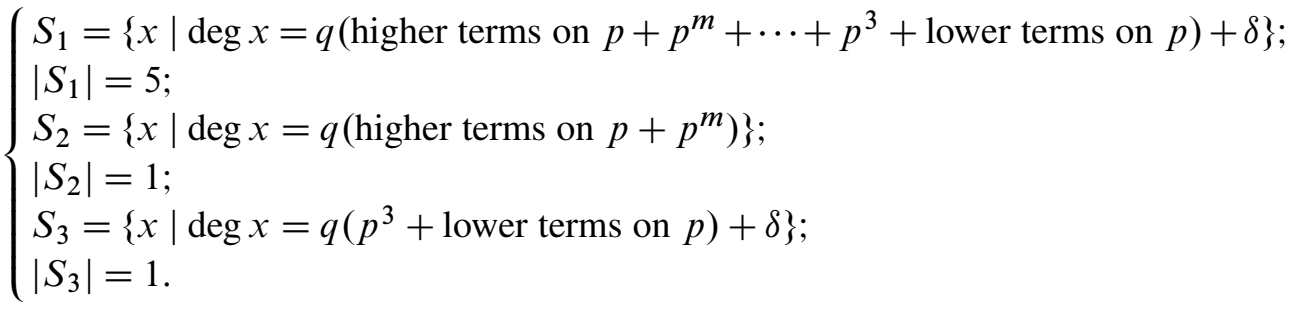

Here $\delta$ may equal 0 or 1 . Now we list the deg's of the seven factors in the following table.

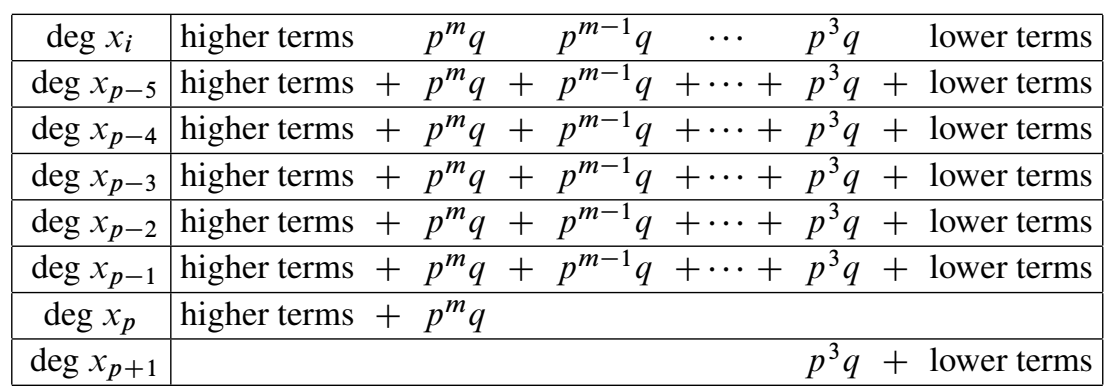

Similarly, from $\sum_{i=p-5}^{p+1} c_{i, m}=6, \sum_{i=p-5}^{p+1} c_{i, m+1}=5, \cdots, \sum_{i=p-5}^{p+1} c_{i, n-1}=5$ and (2.2), we know that there exist at least four factors in $h^{\prime}$ such that

$$
\operatorname{deg} x_{i}=q\left(p^{n-1}+\cdots+p^{m}+\text { lower terms on } p\right)+\delta_{i},
$$

where $\delta_{i}$ may equal 0 or 1 . Then there exist two probabilities which are listed in the following two tables. 


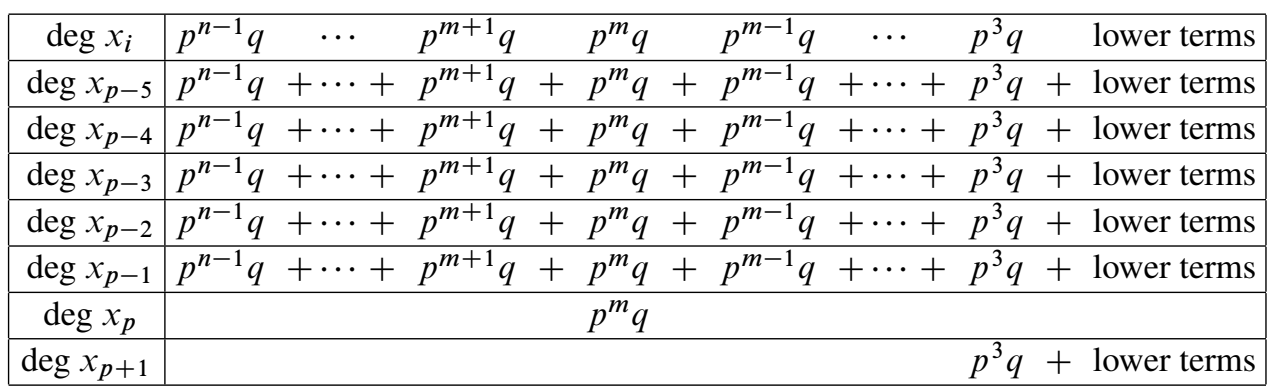

Table 1

It is easy to see that in Table 1 the sixth factor is $h_{1, m}$.

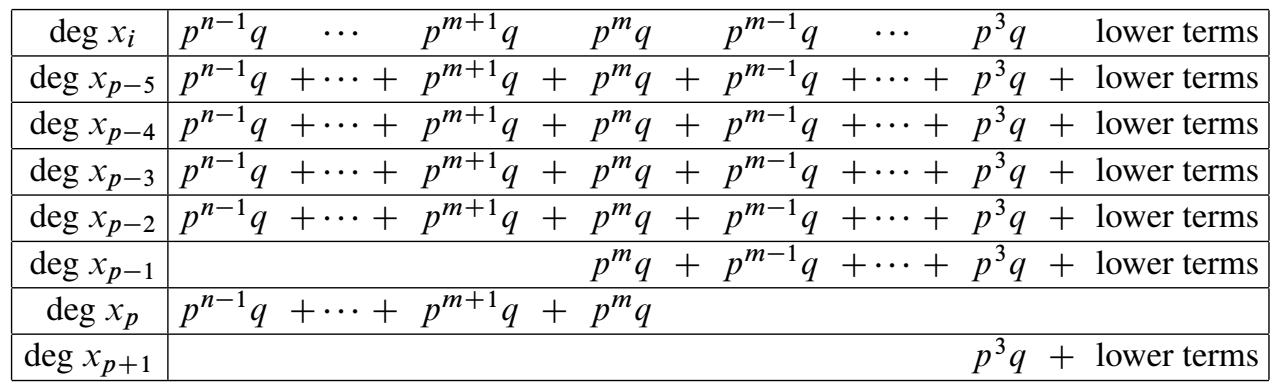

Table 2

It is easy to see that in Table 2 the sixth factor is $h_{n-m, m}$.

Consider Table 1. By $h_{i, j}^{2}=0, \sum_{i=p-5}^{p+1} e_{i}=2, \sum_{i=p-5}^{p+1} c_{i, 0}=4, \sum_{i=p-5}^{p+1} c_{i, 1}=4$, $\sum_{i=p-5}^{p+1} c_{i, 2}=5$ and (2.2), we can get that the deg's of the seven factors of $h^{\prime}$ must be the following.

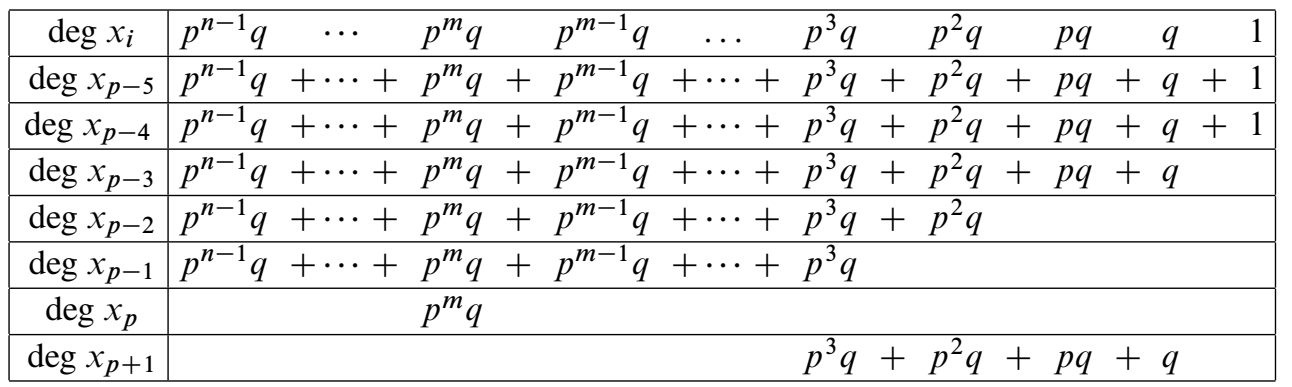

Thus by (2.2), we have that in this case $h^{\prime}=a_{n}^{2} h_{n, 0} h_{n-2,2} h_{n-3,3} h_{1, m} h_{4,0}$. Then up to sign $h=a_{n}^{p-4} h_{n, 0} h_{4,0} h_{n-2,2} h_{n-3,3} h_{1, m} \in E_{1}^{p+1, t(p-4),(2 n+1) p-2 n-9}$, denoted by g1. 
Now consider Table 2. Similarly, we also get $E_{1}^{p+1, t(p-4),(2 n+1) p-2 n-9}$ has seven generators $\mathbf{g} 2, \ldots, \mathbf{g} 8$.

From the above discussion, we get that in this case $E_{1}^{p+1, t(p-4) \text {,* }}$ has the eight generators $\mathbf{g} i(1 \leqslant i \leqslant 8)$.

(ii) When $s=p-5, h=x_{1} x_{2} \cdots x_{l} \in E_{1}^{p+1-r, t(p-5)+1-r, *}$. From the equality $\sum_{i=1}^{l} c_{i, 3}=p$ of (3.2), we have that $l \geqslant p$. Note that $\operatorname{dim} x_{i}=1$ or 2. Since $\operatorname{dim} h=\sum_{i=1}^{l} \operatorname{dim} x_{i}=p+1-r$, it follows that $l \leqslant p+1-r$. Thus we have

$$
p \leqslant l \leqslant p+1-r .
$$

It is easy to see that in this case $r$ is impossible to equal 2 . We only consider the case $r=1$. Thus we have that $l=p$ and $h=x_{1} x_{2} \cdots x_{p} \in E_{1}^{p, t(p-5), *}$. By Theorem 2.4, we know that in this case, it is impossible for $h$ to exist.

Subcase 2.2 $\lambda_{m}=0$. By an argument similar to that used in the proof of $\lambda_{4}=0$ in Case 1 , we also get that in this case

$$
\lambda_{m+1}=0
$$

By induction on $j$, we have that

$$
\lambda_{j}=0 \quad(m+1 \leqslant j \leqslant n-1) .
$$

Thus we have

$$
\left(\lambda_{-1}, \ldots, \lambda_{2}, \lambda_{3}, \ldots, \lambda_{m-1}, \lambda_{m}, \lambda_{m+1}, \ldots, \lambda_{n-1}\right)=(0, \ldots, 0,1, \ldots, 1,0,0, \ldots, 0) \text {. }
$$

By (2.2), it is easy to see that in this case there exists a factor $h_{1, n}$ or $b_{1, n-1}$ in $h$. By the graded commutativity of $E_{1}^{*, *, *}$, we can denote the $h_{1, n}$ or $b_{1, n-1}$ by $x_{l}$. Then $h=h^{\prime \prime} h_{1, n}$ or $h=h^{\prime \prime} b_{1, n-1}$, where $h^{\prime \prime}=x_{1} \cdots x_{l-1}$.

(i) If $x_{l}=h_{1, n}$, then $h^{\prime \prime}=x_{1} \cdots x_{l-1} \in E_{1}^{s+5-r, t(s)-p^{n} q+1-r, *}$. In this case we have $\sum_{i=1}^{l-1} c_{i, 3}=p$. Thus $l \geqslant p+1$. Note that $l \leqslant p+2-r$. It is easy to see that in this case $r$ is impossible to equal 2. Thus we only consider the case $r=1$. Then $l=p+1$. By $\operatorname{dim} x_{i}=2$ or 1 , we have that $\operatorname{dim} h=\operatorname{dim} x_{1} x_{2} \cdots x_{p+1}=s+5 \geqslant p+1$, then $s \geqslant p-4$. Note that $0 \leqslant s<p-3$. It follows that $s=p-4$. Then we have that

$$
h^{\prime \prime}=x_{1} \cdots x_{p} \in E_{1}^{p, t(p-4)-p^{n} q, *} .
$$

By an argument similar to that used in the proof of Subcase 2.1 (i), we get that up to sign $h=a_{m}^{p-4} h_{m, 0} h_{4,0} h_{m-2,2} h_{m-3,3} h_{1, n} \in E_{1}^{p+1, t(p-4),(2 m+1) p-2 m-9}$, denoted by $\mathbf{g} 9$. 
(ii) If $x_{l}=b_{1, n-1}$, then $h^{\prime \prime}=x_{1} \cdots x_{l-1} \in E_{1}^{s+4-r, t(s)-p^{n} q+1-r, *}$. In this case we have $\sum_{i=1}^{l-1} c_{i, 3}=p$. Thus $l-1 \geqslant p$, and then

$$
\operatorname{dim} h^{\prime \prime}=\sum_{i=1}^{l-1} \operatorname{dim} x_{i} \geqslant p .
$$

On the other hand, we also have

$$
\operatorname{dim} h^{\prime \prime}=s+4-r<p+1-r \leqslant p
$$

by $0 \leqslant s<p-3$. This yields a contradiction. Thus in this case, it is impossible for $h$ to exist.

Combining Cases 1 and 2, we complete the proof of the lemma.

Lemma 3.3 Let $p \geqslant 7, n \geqslant m+2>6,0 \leqslant s<p-3$. Then the May $E_{2}$-term

$$
E_{2}^{s+5, t(s), *}=0 .
$$

Here, $t(s)=q\left[p^{n}+p^{m}+(s+3) p^{2}+(s+2) p+(s+2)\right]+s$.

Proof When $0 \leqslant s<p-4$, from Lemma 3.1 we know that in the MSS, $E_{1}^{s+5, t(s), *}=0$. Then we have

$$
E_{2}^{s+5, t(s), *}=0 .
$$

Now we consider the case $s=p-4$. From Lemma 3.1 we have that

$$
E_{1}^{p+1, t(p-4), *}=\mathbb{Z}_{p}\{\mathbf{g} 1, \mathbf{g} 2, \cdots, \mathbf{g} 9\} .
$$

By the first May differential and graded commutativity of $E_{1}^{*, *, *}$, we can easily get that

$$
\begin{aligned}
& d_{1}(\mathbf{g} 1)=-a_{n}^{p-4} h_{n, 0} h_{4,0} h_{n-2,2} h_{1,3} h_{n-4,4} h_{1, m}+\cdots \neq 0, \\
& d_{1}(\mathbf{g} 2)=a_{n}^{p-4} h_{n, 0} h_{m+1,0} h_{n-2,2} h_{1,3} h_{1, m} h_{n-m-1, m+1}+\cdots \neq 0, \\
& d_{1}(\mathbf{g} 3)=a_{n}^{p-4} h_{n, 0} h_{4,0} h_{n-2,2} h_{m-2,3} h_{1, m} h_{n-m-1, m+1}+\cdots \neq 0, \\
& d_{1}(\mathbf{g} 4)=a_{n}^{p-4} h_{n, 0} h_{m+1,0} h_{2,2} h_{n-3,3} h_{1, m} h_{n-m-1, m+1}+\cdots \neq 0, \\
& d_{1}(\mathbf{g} 5)=a_{n}^{p-4} h_{n, 0} h_{4,0} h_{m-1,2} h_{n-3,3} h_{1, m} h_{n-m-1, m+1}+\cdots \neq 0, \\
& d_{1}(\mathbf{g} 6)=a_{n}^{p-5} a_{m+1} h_{n, 0} h_{4,0} h_{n-2,2} h_{n-3,3} h_{1, m} h_{n-m-1, m+1}+\cdots \neq 0, \\
& d_{1}(\mathbf{g} 7)=a_{n}^{p-5} a_{4} h_{n, 0} h_{m+1,0} h_{n-2,2} h_{n-3,3} h_{1, m} h_{n-m-1, m+1}+\cdots \neq 0, \\
& d_{1}(\mathbf{g} 8)=a_{n}^{p-4} h_{m+1,0} h_{4,0} h_{n-2,2} h_{n-3,3} h_{1, m} h_{n-m-1, m+1}+\cdots \neq 0, \\
& d_{1}(\mathbf{g} 9)=-a_{m}^{p-4} h_{m, 0} h_{4,0} h_{m-2,2} h_{1,3} h_{m-4,4} h_{1, n}+\cdots \neq 0 .
\end{aligned}
$$


It is easy to check that $a_{n}^{p-4} h_{n, 0} h_{4,0} h_{n-2,2} h_{1,3} h_{n-4,4} h_{1, m}$ only appears in $d_{1}(\mathbf{g} 1)$, doesn't appear in $d_{1}(\mathbf{g} i)(i \geqslant 2)$. Similarly, we can show that the eight elements

$$
\begin{gathered}
a_{n}^{p-4} h_{n, 0} h_{m+1,0} h_{n-2,2} h_{1,3} h_{1, m} h_{n-m-1, m+1} \\
a_{n}^{p-4} h_{n, 0} h_{4,0} h_{n-2,2} h_{m-2,3} h_{1, m} h_{n-m-1, m+1} \\
\vdots \\
a_{n}^{p-4} h_{m+1,0} h_{4,0} h_{n-2,2} h_{n-3,3} h_{1, m} h_{n-m-1, m+1} \\
a_{m}^{p-4} h_{m, 0} h_{4,0} h_{m-2,2} h_{1,3} h_{m-4,4} h_{1, n}
\end{gathered}
$$

only appear in $d_{1}(\mathbf{g} 2), d_{1}(\mathbf{g} 3), \cdots, d_{1}(\mathbf{g} 8)$ and $d_{1}(\mathbf{g} 9)$ respectively. It follows that $d_{1}(\mathbf{g} 1), d_{1}(\mathbf{g} 2), \cdots, d_{1}(\mathbf{g} 9)$ are linearly independent. Consequently, we have $E_{2}^{p+1, t(p-4), *}=0$.

Theorem 3.4 Let $p \geqslant 7, n \geqslant m+2>6,0 \leqslant s<p-3$. Then the product

$$
h_{0} h_{n} h_{m} \tilde{\gamma}_{s+3} \neq 0 \in \operatorname{Ext}_{A}^{s+6, t(s)}\left(\mathbb{Z}_{p}, \mathbb{Z}_{p}\right),
$$

where $t(s)=q\left[p^{n}+p^{m}+(s+3) p^{2}+(s+2) p+(s+2)\right]+s$.

Proof Since it is known that $h_{1, i}$ and $a_{3}^{s} h_{3,0} h_{2,1} h_{1,2} \in E_{1}^{*, *, *}$ are permanent cycles in the MSS and converge nontrivially to

$$
h_{i}, \quad \tilde{\gamma}_{s+3} \in \mathrm{Ext}_{A}^{*, *}\left(\mathbb{Z}_{p}, \mathbb{Z}_{p}\right)
$$

for $i \geqslant 0$ respectively (see Theorem 1.4),

$$
h_{1,0} h_{1, n} h_{1, m} a_{3}^{s+3} h_{3,0} h_{2,1} h_{1,2} \in E_{1}^{s+6, t(s), *}
$$

is a permanent cycle in the MSS and converges to $h_{0} h_{n} h_{m} \widetilde{\gamma}_{s+3} \in \operatorname{Ext}_{A}^{s+6, t(s)}\left(\mathbb{Z}_{p}, \mathbb{Z}_{p}\right)$.

Case 1 When $0 \leqslant s<p-4$, from Lemmas 3.1 and 3.3 we know that in the MSS

$$
E_{r}^{s+5, t(s), *}=0 \quad(r \geqslant 1) .
$$

Thus the permanent cycle $h_{1,0} h_{1, n} h_{1, m} a_{3}^{s} h_{3,0} h_{2,1} h_{1,2} \in E_{r}^{s+6, t(s), *}$ does not bound and converges to

$$
h_{0} h_{n} h_{m} \tilde{\gamma}_{s+3} \in \mathrm{Ext}_{A}^{s+6, t(s)}\left(\mathbb{Z}_{p}, \mathbb{Z}_{p}\right)
$$

nontrivially in the MSS, ie, $h_{0} h_{n} h_{m} \tilde{\gamma}_{s+3} \neq 0 \in \operatorname{Ext}_{A}^{s+6, t(s)}\left(\mathbb{Z}_{p}, \mathbb{Z}_{p}\right)$.

Case 2 When $s=p-4$, from Lemma 3.3 we have that $E_{2}^{p+1, t(p-4), *}=0$. Thus

$$
E_{r}^{p+1, t(p-4), *}=0 \quad(r \geqslant 2) .
$$


Meanwhile, in the MSS the third degree of $h_{1,0} h_{1, n} h_{1, m} a_{3}^{p-4} h_{3,0} h_{2,1} h_{1,2}$ is $7 p-16$ and none of the third degrees of the generators $\mathbf{g} i(1 \leqslant i \leqslant 9)$ is $7 p-15$. Thus no element hits $h_{1,0} h_{1, n} h_{1, m} a_{3}^{p-4} h_{3,0} h_{2,1} h_{1,2}$ under the May differential $d_{1}$. From the above discussion, $h_{1,0} h_{1, n} h_{1, m} a_{3}^{p-4} h_{3,0} h_{2,1} h_{1,2} \in E_{r}^{p+1, t(p-4), *}$ does not bound in the MSS and converges nontrivially to $h_{0} h_{n} h_{m} \widetilde{\gamma}_{p-1} \in \operatorname{Ext}_{A}^{p+2, t(p-4)}\left(\mathbb{Z}_{p}, \mathbb{Z}_{p}\right)$. Thus $h_{0} h_{n} h_{m} \tilde{\gamma}_{p-1} \neq 0$.

From Cases 1 and 2, Theorem 3.4 follows.

Theorem 3.5 Let $p \geqslant 7, n \geqslant m+2>6,0 \leqslant s<p-3,2 \leqslant r \leqslant s+6$. Then we have

$$
\operatorname{Ext}_{A}^{s+6-r, t(s)-r+1}\left(\mathbb{Z}_{p}, \mathbb{Z}_{p}\right)=0,
$$

where $t(s)=q\left[p^{n}+p^{m}+(s+3) p^{2}+(s+2) p+(s+2)\right]+s$.

Proof From Lemma 3.1, we have that in this case $E_{1}^{s+6-r, t(s)+1-r, *}=0$. By the MSS, we easily have the desired result.

\section{Proof of Theorem 1.5}

From Theorem 1.3, $i_{*}\left(h_{0} h_{n} h_{m}\right) \in \operatorname{Ext}_{A}^{3, q\left(p^{n}+p^{m}+1\right)}\left(H^{*} M, \mathbb{Z}_{p}\right)$ is a permanent cycle in the ASS and converges to a nontrivial element $\xi_{m, n} \in \pi_{q\left(p^{n}+p^{m}+1\right)-3}(M)$.

Consider the composition of maps $\varphi=j j^{\prime} \bar{j} \gamma^{s+3} \bar{i}_{i}^{\prime} \xi_{m, n}$. Since $\xi_{m, n}$ is represented by $i_{*}\left(h_{0} h_{n} h_{m}\right) \in \operatorname{Ext}_{A}^{3, q\left(p^{n}+p^{m}+1\right)}\left(H^{*} M, \mathbb{Z}_{p}\right)$ in the ASS, then the above $\varphi$ is represented in the ASS by $\bar{c}=\left(j j^{\prime} \bar{j} \gamma^{s+3} \bar{i} i^{\prime} i\right)_{*}\left(h_{0} h_{n} h_{m}\right)$.

From Theorem 1.4 and the knowledge of Yoneda products we know that the composition

$$
\begin{aligned}
\operatorname{Ext}_{A}^{0,0}\left(\mathbb{Z}_{p}, \mathbb{Z}_{p}\right) \stackrel{\left(\overline{i i} i^{\prime} i\right)_{*}}{\longrightarrow} \operatorname{Ext}_{A}^{0,0}\left(H^{*} V(2), \mathbb{Z}_{p}\right) \\
\stackrel{\left(j j^{\prime} \bar{j}\right)_{*}\left(\gamma_{*}\right)^{s+3}}{\longrightarrow} \operatorname{Ext}_{A}^{s+3, q\left[(s+3) p^{2}+(s+2) p+(s+1)\right]+s}\left(\mathbb{Z}_{p}, \mathbb{Z}_{p}\right)
\end{aligned}
$$

is a multiplication up to nonzero scalar by

$$
\tilde{\gamma}_{s+3} \in \mathrm{Ext}_{A}^{s+3, q\left[(s+3) p^{2}+(s+2) p+(s+1)\right]+s}\left(\mathbb{Z}_{p}, \mathbb{Z}_{p}\right) .
$$

Hence, $\bar{f}$ is represented up to nonzero scalar by

$$
\bar{c}=\tilde{\gamma}_{s+3} h_{0} h_{n} h_{m} \neq 0 \in \mathrm{Ext}_{A}^{s+6, t(s)}\left(\mathbb{Z}_{p}, \mathbb{Z}_{p}\right)
$$

in the ASS (cf Theorem 3.4). 
Moreover, from Theorem 3.5, we know that $\tilde{\gamma}_{s+3} h_{0} h_{n} h_{m}$ cannot be hit by any differential in the ASS. Consequently, the corresponding homotopy element $\varphi$ is nontrivial. This shows Theorem 1.5.

Acknowledgments The author would like to express his deep thanks to the referee, who read carefully the manuscript of this paper and gave the author many helpful comments and suggestions. The research was partially supported by the NCET, the National Natural Science Foundation of China (Nos. 10501045, 10771105) and the Fund of the Personnel Division of Nankai University.

\section{References}

[1] J F Adams, Stable homotopy and generalised homology, Chicago Lectures in Math., Univ. of Chicago Press (1974) MR0402720

[2] R L Cohen, Odd primary infinite families in stable homotopy theory, Mem. Amer. Math. Soc. 30 (1981) viii+92 MR603393

[3] C-N Lee, Detection of some elements in the stable homotopy groups of spheres, Math. Z. 222 (1996) 231-245 MR1429336

[4] J Lin, Two new families in the stable homotopy groups of sphere and Moore spectrum, Chinese Ann. Math. Ser. B 27 (2006) 311-328 MR2239830

[5] X Liu, A nontrivial product in the stable homotopy groups of spheres, Sci. China Ser. A 47 (2004) 831-841 MR2127211

[6] A Liulevicius, The factorization of cyclic reduced powers by secondary cohomology operations, Mem. Amer. Math. Soc. No. 42 (1962) 112 MR0182001

[7] H R Miller, D C Ravenel, W S Wilson, Periodic phenomena in the Adams-Novikov spectral sequence, Ann. Math. (2) 106 (1977) 469-516 MR0458423

[8] D C Ravenel, Complex cobordism and stable homotopy groups of spheres, Pure and Applied Math. 121, Academic Press, Orlando, FL (1986) MR860042

[9] L Smith, On realizing complex bordism modules. Applications to the stable homotopy of spheres, Amer. J. Math. 92 (1970) 793-856 MR0275429

[10] H Toda, On spectra realizing exterior parts of the Steenrod algebra, Topology 10 (1971) 53-65 MR0271933

School of Mathematical Sciences and LPMC, Nankai University Tianjin 300071, PR China

xgliu@nankai.edu.cn, matlxg@gmail.com

Received: 24 May 2007 Revised: 10 December 2008 